\title{
Pensionskasse als Instrument der Steueroptimierung
}

Urs Gross

Korrespondenz:

Urs Gross, Unternehmensberater

Comes Treuhand AG

FMH Treuhand Services

Ackeretstrasse 3

Postfach 1659

CH-8401 Winterthur

urs.gross@fmhtreuhand.ch
Der selbständige Unternehmer, also auch der praktizierende Arzt, ist bekanntlich gesetzlich nicht verpflichtet, sich der beruflichen Vorsorge im Sinne des BVG anzuschliessen. In der Praxis wird den Ärzten vielfach geraten, eine gebundene Vorsorgepolice 3a abzuschliessen. Dabei kann der Arzt 20\% seines Ertrages, maximal Fr. 30 960.-, steuerlich in Abzug bringen, sofern er diesen Betrag bis Ende Jahr bei der Versicherungsgesellschaft einzahlt. Diese Lösung hat, nebst dem genannten Vorteil der Steuerabzugsfähigkeit, einige Nachteile:

- der Ertrag des Arztes kann jährlich sehr unterschiedlich sein, weshalb die Berechnung des Einzahlungsbetrages schwierig ist;

- bei einem Einkommen über Fr. $150000 .-$ genügt diese Lösung nicht, es entstehen keine Vorsorgelücken analog der 2. Säule.

Empfehlenswert ist es deshalb auch für den selbständig tätigen Arzt, sich einer Vorsorgeeinrichtung der 2. Säule anzuschliessen. Nicht nur die Beiträge können in der Erfolgsrechnung als Aufwand abgesetzt werden. Es entstehen sogenannte Vorsorgelücken, in welche sich der Arzt einkaufen kann. Die Ärzte können sich ihr Einkaufspotential selber erhöhen. Je höher der versicherte Lohn, desto höher steigt die Vorsorgelücke. Gemäss der Gesetzesänderung, die per 1. Januar 2006 in Kraft trat, ist das versicherbare Einkommen auf das Zehnfache des BVG-Obligatoriums begrenzt, nämlich auf Fr. 774 000.-.

Einkäufe in die Pensionskasse erhöhen die Rente und sind die einfachste und wohl im heutigen Zeitpunkt die wichtigste Möglichkeit der Steueroptimierung. Sind diese Einkäufe aus dem Privatvermögen finanziert, können sie vom steuerbaren Einkommen abgezogen werden. Die Steuerlast wird je nach Einkommenssituation merklich vermindert. Zudem werden die Erträge nicht besteuert und die Vermögenssteuer entfällt.

Nicht ganz so einfach ist die Berechnung des Einkaufspotentials. Jeder Versicherte erhält jährlich einen Leistungsausweis. In einigen Leistungsausweisen ist die mögliche Einkaufssumme bereits angegeben. Andernfalls wird die Pensionskasse die entsprechenden Auskünfte kostenlos erteilen. Zu berücksichtigen sind dann allerdings auch allfällige Guthaben auf Freizügigkeitskonten resp. -policen. Einige Regeln sind jedoch auch hier zu beachten:

- Vorbezüge für Wohneigentum, sogenannte WEF-Bezüge, müssen zuerst zurückbezahlt werden, bevor erneut Einkäufe getätigt werden können;

- eingekaufte Beträge können in den folgenden Jahren nicht in Kapitalform bezogen werden (ab Alter $60 \mathrm{zu}$ beachten);

- wer aus dem Ausland in die Schweiz zieht, kann in den ersten fünf Jahren nur maximal $20 \%$ des versicherten Lohnes einkaufen.

Sofern ein Arzt bereits die eingangs erwähnte Vorsorgepolice 3a abgeschlossen hat, kann diese ohne weitere Probleme in die Pensionskasse überführt werden. Selbstverständlich ist dieser Betrag steuerlich nicht mehr absetzbar. Er verbessert dagegen die Situation bei den Rentenleistungen.

Es lohnt sich auf jeden Fall, die gesamte Vorsorgesituation unter dem Aspekt der Steueroptimierung von einem Spezialisten überprüfen zu lassen. 